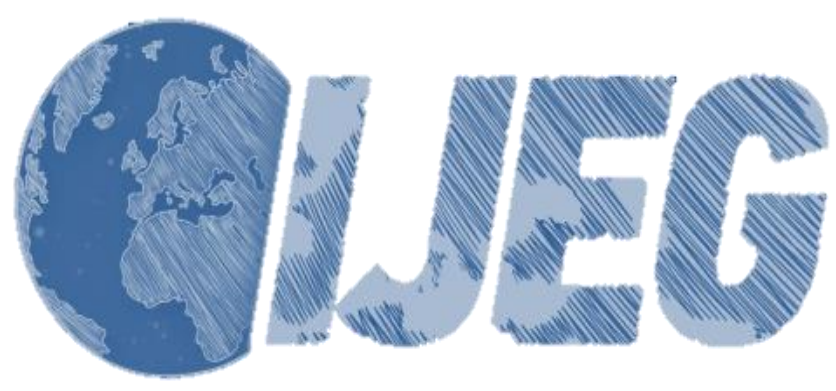

International Journal of Engineering and Geosciences (IJEG), Vol; 4, Issue; 2, pp. 078-087, June, 2019, ISSN 2548-0960, Turkey, DOI: 10.26833 ijeg. 455595

\title{
OBJECT BASED BURNED AREA MAPPING WITH RANDOM FOREST ALGORITHM
}

\author{
Resul Comert ${ }^{1}$, Dilek Kucuk Matc1 ${ }^{2 *}$, Ugur Avdan ${ }^{2}$ \\ ${ }^{1}$ Gumushane University/Faculty of Engineering And Natural Sciences/Department Of Geomatic Engineering/ \\ Gümüşhane, Turkey (rcomert@gumushane.edu.tr); ORCID 0000-0003-0125-4646; \\ ${ }^{2}$ Institute of Space and Earth Sciences, Eskişehir Technical University, Eskişehir, Turkey, Turkey
} (dkmatci@ anadolu.edu.tr, uavdan@eskisehir.edu.tr); ORCID 0000-0002-4078-8782; ORCID 0000-0001-7873-9874;

*Corresponding Author, Received: 28/08/2018, Accepted: 05/02/2019

\begin{abstract}
It is very important to map the burned forest areas economically, quickly and with the high accuracy of issues such as damage assessment studies, fire risk analysis, and management of forest regeneration processes. Remote sensing methods give advantages such as fast, easy-to-use and high accuracy for burned area mapping. Recent years machine learning algorithms have become more popular in satellite image classification, due to the effective solutions for the analysis of complex datasets which have a large number of variables. In this study, the success of object based random forest algorithm was investigated for burned forest area mapping. For this purpose, Object based image analysis (OBIA) was performed using Landsat 8 image of the Adrasan and Kumluca fires which occurred in $24-27$ June 2016 . The study consisted of five steps. In the first step, the multi-resolution image segmentation was performed for obtaining image objects from Landsat 8 spectral bands. In the second step, the image object metrics such as spectral index and layer values were calculated for all image objects. In the third step, a random forest classifier model was developed. Then, the developed model applied to the test site for classification of the burned area. Finally, the obtained results evaluated with confusion matrix based on the randomly sampled points. According to the results, we obtained 0.089 commission error (CE) with 0.014 omission error $(\mathrm{OE})$. An overall accuracy was obtained as 0.99 . The results show that this approach is very useful to be used to determine burned forest areas.
\end{abstract}

Keywords: Random forest, Burned area mapping, Object based image analysis, Remote sensing 


\section{INTRODUCTION}

Forests, one of the most important natural sources, provide a rich biodiversity that contributes directly and indirectly to economic and social life. The destruction of forests by fire leads to negative effects such as environmental pollution, destruction of historical and natural wealth, picnic places, forest parks, hunting areas and sports areas that are suitable for the city life and adversely affecting the economy (Adams, 2013).

Especially for the countries located in the Mediterranean climate zone, forest fires are among the most important natural threats (Hernandez, Drobinski, \& Turquety, 2015). About 50,000 fires occur each year in these countries and about 700,000-1,000,000 ha of forest area are exposed to fire (Dimitriou, Mantakas, \& Kouvelis, 2001). Mapping burned areas can determine the effects of fires (G. Chen et al., 2017; Meng et al., 2017; Palandjian, Gitas, \& Wright, 2009) and help to plan and manage operations to prevent desertification, biodiversity loss, flooding and soil erosion, which can occur after a fire (Vallejo, Arianoutsou, \& Moreira, 2012). It can also be used as input data for the generation of fire risk maps of the future(Filippidis \& Mitsopoulos, 2004).

Satellite remote sensing has offered great advantages in the monitoring and mapping of burned areas since the 1980s (Flannigan \& Haar, 1986). Optical satellite data has been especially successful in generating a burned area inventory on the continental scale (Barbosa, Grégoire, \& Pereira, 1999), regional scale (Giglio, Loboda, Roy, Quayle, \& Justice, 2009; Loboda, O'neal, \& Csiszar, 2007) and national scale(Palandjian et al., 2009). Many image analysis techniques, such as vegetation and burn index (Chuvieco, Martin, \& Palacios, 2002; Epting, Verbyla, \& Sorbel, 2005; Escuin, Navarro, \& Fernandez, 2008; Loboda et al., 2007; Pereira, 1999), supervised classification (Palandjian et al., 2009), logistic regression (Bastarrika, Chuvieco, \& Martín, 2011), spectral angle mapper and artificial neural network (Petropoulos, Vadrevu, Xanthopoulos, Karantounias, \& Scholze, 2010), Neuro-fuzzy (Mitrakis, Mallinis, Koutsias, \& Theocharis, 2012) and support vector machine (Petropoulos, Kontoes, $\&$ Keramitsoglou, 2011), have been successfully applied to pixel based satellite data of various resolutions.

Pixel based image analysis (PBIA) and object based image analysis (OBIA) techniques are the two main image analysis approach in satellite image classification. While PBIA approach works on each individual pixel for extracting information from satellite images, OBIA approach uses image objects that consist of homogenous pixel groups. While pixel based approach has generally applied to medium and low spatial resolution images, OBIA has applied to high and very high spatial resolution images. There were many studies that applied to OBIA to medium and low resolution images for burned area mapping (Gitas, Mitri et al. 2004, Polychronaki and Gitas 2012, Katagis, Gitas et al. 2014, Kavzoglu, Erdemir et al. 2016), (Gitas, Mitri et al. 2004). Analyzing the studies using medium resolution satellite images to compare these two approaches, OBIA gives more accurate results than PBIA (Estoque, Murayama, \& Akiyama, 2015; Gao, Mas, Kerle, \& Pacheco, 2011; Gilbertson, Kemp, \& van Niekerk, 2017; Varamesh, Hosseini, \& Rahimzadegan, 2017). Also, OBIA reduce the salt and pepper effect that cause misclassified pixel on satellite images (Phiri \& Morgenroth, 2017)(Gao et al. 2011). For these reasons,
OBIA was selected for burned forest area mapping in this study.

Object-based classification of burned areas has been applied to very high-resolution images (Dragozi, Gitas, Stavrakoudis, \& Theocharis, 2014), high-resolution images (Sertel \& Alganci, 2016), medium resolution images (Katagis, Gitas, \& Mitri, 2014; Kavzoglu, Erdemir, \& Tonbul, 2016; Mitri \& Gitas, 2004; Polychronaki \& Gitas, 2012), low resolution images (Gitas, Mitri, \& Ventura, 2004) and SAR images(Polychronaki, Gitas, Veraverbeke, \& Debien, 2013). The OBIA has two main steps, segmentation and classification (Baatz, Hoffmann, \& Willhauck, 2008). Multi-resolution segmentation used in the segmentation phase is a preferred method (Benz, Hofmann, Willhauck, Lingenfelder, \& Heynen, 2004). The classification process is carried out by rule-based or supervised classification.

In many studies, rule-based classification methods have been used to map burned areas along with objectbased classification methods. The rule-based classification has two limitations, although it does not yield successful results in the removal of burned areas. These are, (i) the difficulty in deciding which descriptive properties are really important within a large number of object metrics in large data sets, and (ii) its limited applicability to different environmental conditions and different data types (Stumpf \& Kerle, 2011). Therefore, in the extraction of burned fields from complex datasets and data sets with a large number of variables, there is a need to implement other classification algorithms. Machine learning algorithms such as random forest (Breiman, 2001) provide effective solutions for the analysis of complex datasets. Random forest has been successfully applied to areas such as mapping landslides (Breiman, 2001; W. Chen, Li, Wang, Chen, \& Liu, 2014; Stumpf \& Kerle, 2011), gene selection (Díaz-Uriarte \& De Andres, 2006), land cover classification (Gislason, Benediktsson, \& Sveinsson, 2006) and hyperspectral image classification (Ham, Chen, Crawford, \& Ghosh, 2005). Also, it has been used forest fire studies such as fire occurrence modeling (Gislason et al., 2006), forest and woodland severity analysis (Dillon et al., 2011; Holden, Morgan, \& Evans, 2009). There is only one study is available in the literature for the mapping of burned areas with the random forest based classifier. This classifier was developed to extract the burned areas on the global scale from the MODIS images (Ramo \& Chuvieco, 2017).

In this study, we evaluated the performance of the random forests algorithm for mapping of burned forest areas from remotely sensed images that were segmented to image objects. This approach allows the advantages of an object-based classification method to combine random forest algorithm with more accurate extraction of burned areas.The images of the Kumluca and Adrasan regions (the study area) are obtained for free from the Landsat 8 satellite. First, the images are segmented using multiresolution segmentation for obtaining image objects. After the image object attributes such as band indices and band value were calculated, training and test datasets were generated. The training data were used to determine the optimal random forest classification model by testing different parameter and attribute importance for best result is calculated. The classification model was applied to test data set. The accuracy level of the results was 
evaluated according to the confusion matrix based on the randomly sampled points. Also, the map obtained from the local authority and the map obtained by the proposed method were compared according to the total burned areas. In addition, Normalize Difference Vegetation Index (NDVI) based change detection map was used for interpretation of results. The results show that the random forest algorithm has the potential to be used as a tool by forest management authorities to identify burned forest areas with high accuracy and low cost.

\section{STUDY AREA AND DATA SETS}

In this study, the forest fires that occurred on 24-27 June 2016 in Adrasan and Kumluca regions in Antalya province were investigated (Figure 1). The province of Antalya is located in the Mediterranean climate zone, where there is a risk of first-degree fire. The existing forest area is $1,146,062$ hectares, covering $56 \%$ of the province's surface area. The forest areas in the province correspond to $5.4 \%$ of the forest areas of Turkey. The tree species in the Antalya forest area are composed of the red leaf $(65 \%)$, cedar $(16 \%)$, black cherry $(8 \%)$, fir $(5 \%)$, juniper (4\%) and other leafy species (URL 1). During the fire that occurred on $24-27$ June 2016, very large forest areas were destroyed, animals, greenhouses and houses were badly affected (Neyisci, Sirin, Bas, \& Saribasak, 2016).
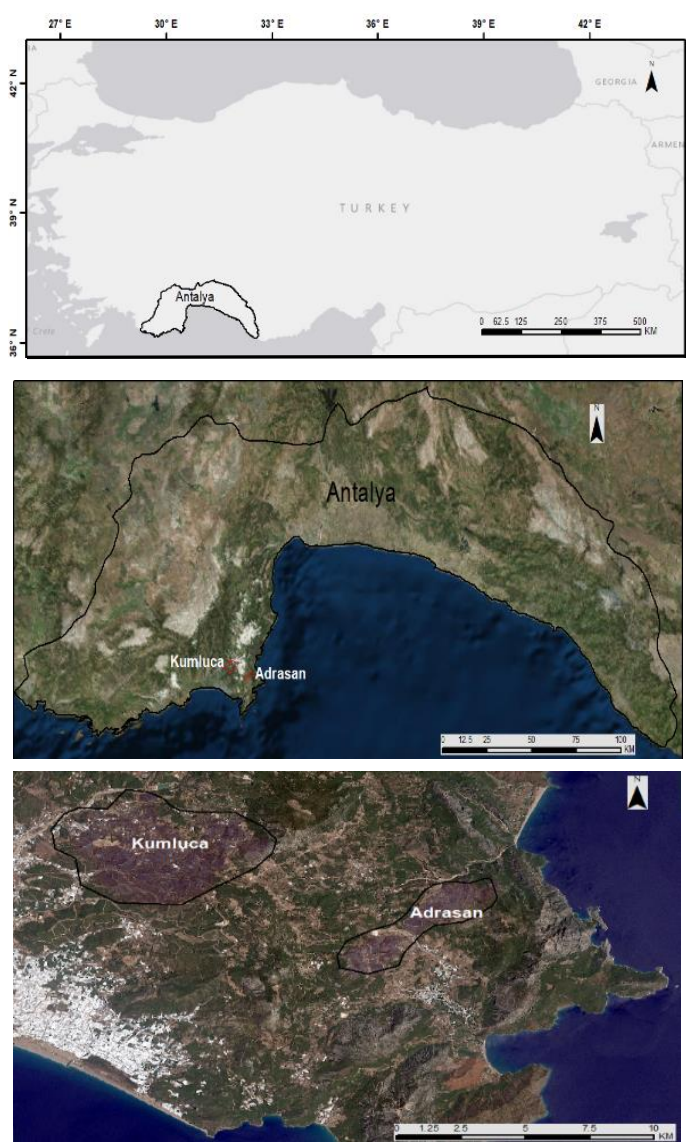

Figure. 1 Location of Adrasan and Kumluca

Landsat 8 Operational Land Imager (OLI) L1TP postevent (08 July 2016) data were used in this study. The
Landsat L1TP collection is radiometrically calibrated and orthorectified using ground control points and digital elevation model (DEM) data, to correct for relief displacement (URL 2). Post-event image bands were used for mapping the area with random forest. Landsat 8 OLI sensor images consist of nine spectral bands. Excluding the cirrus and ultra-blue bands, seven bands were used for burned area analysis (Table 1). Also, pre-event image (which was taken 22 June 2016) was utilized for obtaining NDVI based change detection map.

Table 1 Landsat 8 OLI bands, wavelengths and image resolutions

\begin{tabular}{ccc}
\hline Band & $\begin{array}{c}\text { Wavelength } \\
\text { (micrometers) }\end{array}$ & $\begin{array}{c}\text { Resolution } \\
\text { (meters) }\end{array}$ \\
\hline Band 2 & $0.452-0.512$ & 30 \\
Band 3 & $0.533-0.590$ & 30 \\
Band 4 & $0.636-0.673$ & 30 \\
Band 5 & $0.851-0.879$ & 30 \\
Band 6 & $1.566-1.651$ & 30 \\
Band 7 & $2.107-2.294$ & 30 \\
Band 8 & $0.503-0.676$ & 15 \\
\hline
\end{tabular}

\section{METHODS}

In this study, applied methods was composed of five steps which were pre-processing, image segmentation, calculation of image object attributes, classification and accuracy assessment.

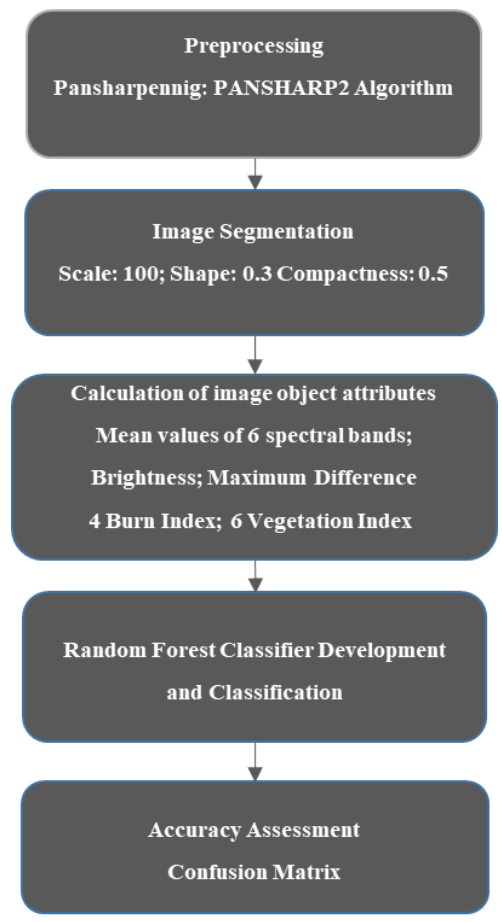

Figure. 2 Flowchart of the methodology for burned area classification 


\subsection{Preprocessing}

During the preprocessing phase, 30-meter spectral bands were pansharpened with a panchromatic band of the 15 - meter resolution. In this phase, the PANSHARP2 algorithm in PCI Geomatica (2016) software was used for fusion of panchromatic and spectral bands. This versatile and extremely simple algorithm that can work with any data type is based on the least squares and a statistical approach. The first step in the PANSHARP 2 algorithm is to co-register the panchromatic band and multispectrum image bands together as geographically corrected. This algorithm attempts to protect spectral properties, mean, standard deviation, and histogram shape for each channel. When calculating the best gray value with the smallest squares and color presentation, statistical approximation and automatic fusion were performed (Zhang, 2002).

\subsection{Image Segmentation}

In this study, the advantages of the object-based classification method were used to obtain a more accurate result. This method has been used in the literature to overcome the limitations and weaknesses of pixel based image analysis.

The first stage of object-based classification is the creation of homogeneous and meaningful image objects for image segmentation. Over recent decades, a number of image segmentation methods have been developed for remote sensing image analysis (Dey, Zhang, \& Zhong, 2010). In this study, the multi-resolution segmentation (MRS) method was used. MRS is a region enhancement algorithm that combines pixels or existing image objects together. The method starts at the pixel level and combines neighboring pixels depending on a spectral and geometric homogeneity criterion. In the study, MRS was implemented using Ecognition Developer (version: 9.0) software. In the segmentation process, 6 spectral bands, which are pansharpened to $15 \mathrm{~m}$, were used. In order to obtain optimal image objects, the scale parameter, shape, integrity and layer weight were specified by the user (Benz, Hofmann, Willhauck, Lingenfelder, \& Heynen, 2004). As a result of a visual analysis made by the trial and error method, the appropriate parameter values for the data set were determined as scale factor 100, shape 0.3 and compactness 0.5 . The equal weight values were assigned to all bands.

\subsection{Calculation of Image Object Attributes}

The forest fires directly affect the vegetation. There are many studies on burned area mapping and burned severity assessment use burned area indices and vegetation indices for increasing success of the methods (Chuvieco et al., 2002; Fraser, Li, \& Cihlar, 2000; Loboda et al., 2007; Schepers et al., 2014). Starting from that, commonly used indices and other band values were preferred object image attributes. Eighteen object attributes were calculated for use in the classification steps of random forest, described below:

The mean values of six spectral bands (Blue, Green, Red, NIR, SWIR 1, SWIR 2), defined as the average of the reflection values of the pixels that form an image object.

The mean brightness (B) calculated as the sum of the object means in the bands $\left.\overline{(}_{i(v i s)}\right)$ divided by the number of the corresponding bands $\left(\mathrm{n}_{\mathrm{vis}}\right)$ (Stumpf \& Kerle, 2011):

$$
B=\left(1 / n_{v i s}\right) \sum_{i=1}^{n_{v i s}} \bar{c}_{i(v i s)}
$$

The maximum difference, defined as the absolute value of the difference between the minimum object mean $\min \left(\bar{C}_{i(v i s)}\right)$ and maximum object mean $\max \left(\bar{C}_{i(v i s)}\right)$, divided by the object brightness B (Stumpf \& Kerle, 2011):

$$
\underset{B}{\operatorname{MaxDiff}}=\left(\max \left(\bar{c}_{i(v i s)}\right)-\min \left(\bar{c}_{i(v i s)}\right)\right) /
$$

Four burned area indexes, as frequently referred to in the literature, calculated for image objects: burned area index (BAI), normalized burn ratio (NBR) (Key \& Benson, 2006), normalized burn ratio 2 (NBR2), and midinfrared burn index (MIRBI) (Trigg \& Flasse, 2001):

$$
\begin{aligned}
& B A I=1 /\left((0.1-R e d)^{2}+(0.06-N I R)^{2}\right) \\
& N B R=(N I R-S W I R 2) /(N I R+S W I R 2) \\
& \text { NBR2 }=(\text { SWIR } 1-\text { SWIR2 }) /(\text { SWIR1 }+ \\
& \text { SWIR2 })
\end{aligned}
$$$$
M I R B I=10 S W I R 2-9.8 S W I R 1+2
$$

Six spectral indices for burned area and fire damage detection, the normalized difference vegetation index (NDVI) (Tucker, 1979), global environmental monitoring index (GEMI) (Pinty \& Verstraete, 1992), enhanced vegetation index (EVI)(A. Huete et al., 2002), soiladjusted vegetation index (SAVI) (A. R. Huete, 1988), char Soil Index (CSI) (Smith et al., 2007) and normalized difference moisture index (NDMI) (Wilson \& Sader, 2002). The equation for:

$$
\begin{array}{r}
\text { NDVI }=(\text { NIR }- \text { RED }) /(\text { NIR } \\
+ \text { RED })
\end{array}
$$

GEMI $=\gamma(1-0.25 \gamma)-(\operatorname{RED}-0.125) /(1-\mathrm{RED})$

with

$\gamma=\left((2 \mathrm{NIR})^{2}-\mathrm{RED}^{2}+1.5 \mathrm{NIR}+0.5 \mathrm{R}\right) /$

$(\mathrm{NIR}+\mathrm{RED}+0.5)$

EVI $=2.5 *(($ NIR - RED $) /($ NIR -6 RED 7. 5BLUE + 1))

$\mathrm{SAVI}=(1+\mathrm{L})((\mathrm{NIR}-\mathrm{RED}) /(\mathrm{NIR}+\mathrm{RED}+\mathrm{L}))$

with 
$\mathrm{L}=0.5$

CSI $=$ NIR/SWIR2

$\mathrm{NDMI}=(\mathrm{NIR}-\mathrm{SWIR} 1) /(\mathrm{NIR}+\mathrm{SWIR} 1)$

\subsection{Classification}

The random forest method, which is a machine learning algorithm, is used for the classification of the burned areas. The random forest method, developed by Breiman (2001), is a nonparametric mass learning algorithm that uses numerous decision trees in the classification process (Breiman, 2001). According to Breiman (Breiman, 1996), with a classification process using a single decision tree, small changes in training lead to high variance. And this situation reduces the accuracy of classification. Random forest, on the other hand, forms a multi-decision tree with sub-datasets randomly selected in the training data. Each decision tree is voted for, according to its class membership, and the decision tree that receives the most votes is used in predicting the related class (Stumpf \& Kerle, 2011).

The random forest algorithm works according to the supervised classification method. In this context, training and test data are needed. Within the scope of the study, the Kumluca region was selected as the training area and the Adrasan region as the test area. In the training area, image segments were defined in two classes, burned area (BA) and non-burned area (NBA). There are 13551 number of the image objects in the training data field. The 501 number of them were burned areas, and the others were non-burned areas. There are 9968 image segments in the test data (Figure 3).

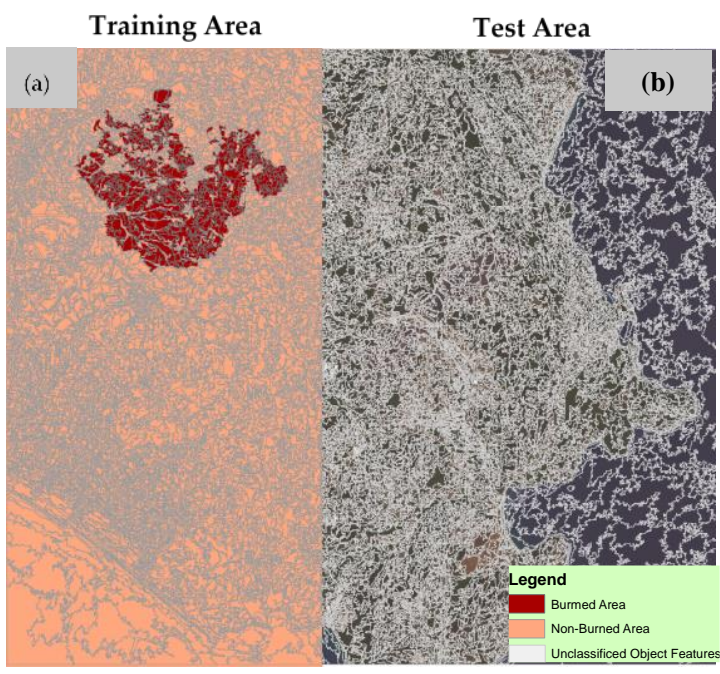

Figure 3. (a) Kumluca training area included 501 burned areas (BA) image objects and 13050 non-burned area image objects; (b) Adrasan test site included 9968 unclassified image objects

In the study, the model parameters to be used in the classification of the training data were determined first. In the random forest algorithm, there are two parameters must be determined by the user. These are the number of trees $(\mathrm{N})$ and the number of attributes to choose randomly (m). These two parameters were applied to the training data with various values, in order to find the appropriate model values for classification. The $\mathrm{m}$ value was generally calculated using $V_{\mathrm{m}}$ equation. 20 different random forest classifier models which were a combination of the ten numbers of trees $(100-1000$ increase by 100) and two numbers of attributes $(\mathrm{m}=4,5$ which indicate integer value the lower and upper values close to $\sqrt{\mathrm{m}}$ ) was built for determining the optimum parameter. The result was evaluated Balanced Accuracy (Kuhn \& Johnson, 2013; Ramo \& Chuvieco, 2017) defined in Table 2.

Balanced Accuracy $=\left(\frac{\mathrm{E} 11}{(\mathrm{E} 11+\mathrm{E} 21)}+\frac{\mathrm{E} 22}{(\mathrm{E} 22+\mathrm{E} 12)}\right) / 2$

Table 2. Structure of Burned Area Confusion Matrix

\begin{tabular}{cccc}
\hline \multicolumn{4}{c}{ Reference } \\
\hline \multirow{2}{*}{ Predicted } & \multirow{2}{*}{ Burned } & $\begin{array}{c}\text { Non }- \\
\text { Burned }\end{array}$ & $\begin{array}{c}\text { Row } \\
\text { Total }\end{array}$ \\
\hline Burned & $\mathrm{E}_{11}$ & $\mathrm{E}_{12}$ & $\mathrm{E}_{1+}$ \\
Non - Burned & $\mathrm{E}_{21}$ & $\mathrm{E}_{22}$ & $\mathrm{E}_{2+}$ \\
Col. Total & $\mathrm{E}_{+1}$ & $\mathrm{E}_{+2}$ & $\mathrm{E}_{\Sigma}$ \\
\hline
\end{tabular}

\subsection{Accuracy Assessment}

The random forest classification results were validated using a confusion matrix, known as the most common method of image classification in remote sensing applications. It was possible to calculate overall accuracy, omission errors and commission errors using the confusion matrix (Banko, 1998). Classification results which applied to test data evaluated two different ways. First, 650 random points were generated for evaluation of the classification results. Then the result was compared local authority map. Following equation shows error value calculations. A parameter which is used in equation same as the Table 2.

$$
\begin{aligned}
& \text { Omission Error }=\left(E_{+1}-E_{11}\right) / E_{+1} \\
& \text { Commission error }=\left(E_{1+}-E_{11}\right) / E_{1+} \\
& \text { Overall Accuracy }=\left(E_{11}-E_{22}\right) / E_{\Sigma}
\end{aligned}
$$

\section{RESULTS}

\subsection{Optimum parameter selection for Random Forest (RF)}

Developing of random forest classifier, open source WEKA Data Mining software was used for burned forest area mapping. random forest algorithm was applied to the training data as ten-fold cross-validation procedure. In this purpose, the data were randomly divided into ten parts. Nine parts are used as training data and one part is used as validation data. This step continues in a way that all parts are applied alternately. The 20 different classifier model was developed according to two attribute's value 
$(4,5)$ and ten different numbers of trees (100-1000 increase by 100). Figure 4 shows the balanced accuracy of 20 models according to the different number of randomly selected attributes $(\mathrm{m})$ and the number of trees $(\mathrm{N})$. It was also tested in other values that are different from the attribute numbers 4 and 5. The similar results were obtained for other numbers of attributes. Therefore, only 4 and 5 attributes value result is shown in Figure 4. According to Figure optimum parameters were selected as $\mathrm{N}=400$ and $\mathrm{m}=5$ which give higher balanced accuracy (0.914).

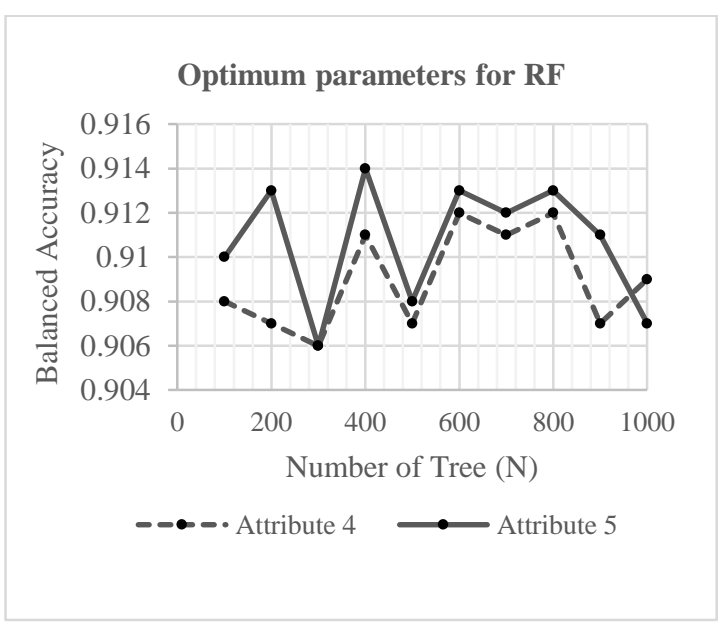

Figure 4. Balanced Accuracy value for different number of randomly selected attributes and number of trees.

\subsection{Attributes Importance}

The spectral bands in remote sensed images and spectral indices obtained with the help of these bands provide a wide range of variable. The use of these detailed variable increases the processing time as much information does not always provide the right result. Therefore, it should be determined which features are important for classification to reduce the data size.

The attribute importance was calculated with the different methods in the literature (Biau, Devroye, \& Lugosi, 2008; Ishwaran, 2007; Louppe, Wehenkel, Sutera \& Geurts, 2013; Meinshausen, 2006). In their study, Louppe at.all (2013) indicate that the average impurity decrease value for each input variable is equal to zero only if the variable is irrelevant.

In this study attribute importance was calculated according to the average impurity decrease for selected optimum random forest parameters (Louppe et al., 2013). Figure 5 shows the importance of every attribute which was used in this study. All attributes have very closed importance each other. But NDVI has seen the most important attributes which have 0.34 impurity decrease. GEMI and EVI indices that have 0.32 impurity decrease are secondly important attributes. According to attributes importance results there is no variable that equal to zero. Therefore, all attributes were used for classification.

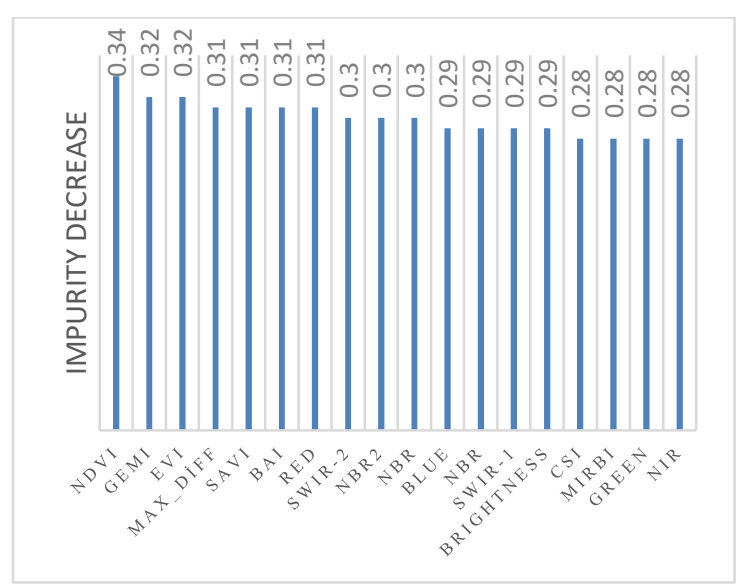

Figure 5. Attribute importance based on the average impurity decrease.

\subsection{Random Forest Classification Results for Adrasan Test Site}

The developed random forest classifier model for training data based on optimum parameters was applied the test dataset. The obtained results were evaluated with two different ways. First, randomly 650 points were generated on a test site to evaluate the classification results. Then, obtained map compare with local authority map.

According to the random points results, the object based random forest classification achieves 0.089 commission error (CE) with 0.014 omission error (OE). An overall accuracy was obtained as 0.99 . Table 3 shows the error matrix with descriptive statistics.

Table 3. Accuracy assessment using 650 random points for object-based random forest classification, overall accuracy (OA), omission error (OE) and commission error (CE) estimated based on confusion matrix. (BA: Burned Area; NBA: Non-Burned Area)

\begin{tabular}{cccccc}
\hline \multicolumn{7}{c}{ Reference } \\
\hline Classification & BA & NBA & TOTAL & CE & OE \\
BA & 71 & 7 & 78 & 0.089 & 0.014 \\
NBA & 1 & 571 & 572 & 0.017 & 0.012 \\
Total & 72 & 578 & 650 & & \\
\hline \multicolumn{7}{c}{ Overall Accuracy } & 0.987 \\
\hline
\end{tabular}

The random forest classification result compared with a local authority map which was generated by the Antalya Forest General Directorate. Table 4 shows evaluation results. According to the Table 4, the total area of the burned forest is in the map obtained from local authority 609.48 ha, in the result map of the classification 483.98 ha. The difference between them is 125.5 ha. 
Table 4. Comparison of the result of random forest classification and the map obtained from local authority (unit: hectares)

\begin{tabular}{lcc}
\hline $\begin{array}{l}\text { Local } \\
\text { Map }\end{array}$ & $\begin{array}{l}\text { Random Forest } \\
\text { Classification }\end{array}$ & Difference \\
\hline 609.48 & 483.98 & 125.5 \\
\hline
\end{tabular}

\section{DISCUSSION}

In the segmentation process, the similar pixels were grouped based on the object-based classification method This process reduced the number of input data for classification process. in the analysis to ensure quick results. In the study, after the segmentation process the number of the input data to be classified decreased from 1762725 to 23519 .
The random forest algorithm is a fast-running classification method that can be applied to a large number of data and variables. In the study, burned areas were successfully determined using the 18 variables. Since the variables were randomly selected in the classification process with the random forest algorithm, in each classification, different variables could be included. Also, which attributes are important for classification could be calculated.

In this study, a random forest classifier model was developed for Landsat 8 data. The developed model was tested on Adrasan burned area. Evaluation of results two different reference data which area 650 random points and the local authority map was used. Ground truth for 650 random points was labeled using the RGB color map of Landsat 8, Google Earth images and NDVI difference maps. 650 random points results give higher accuracy with 0.089 commission and 0.014 omission error.

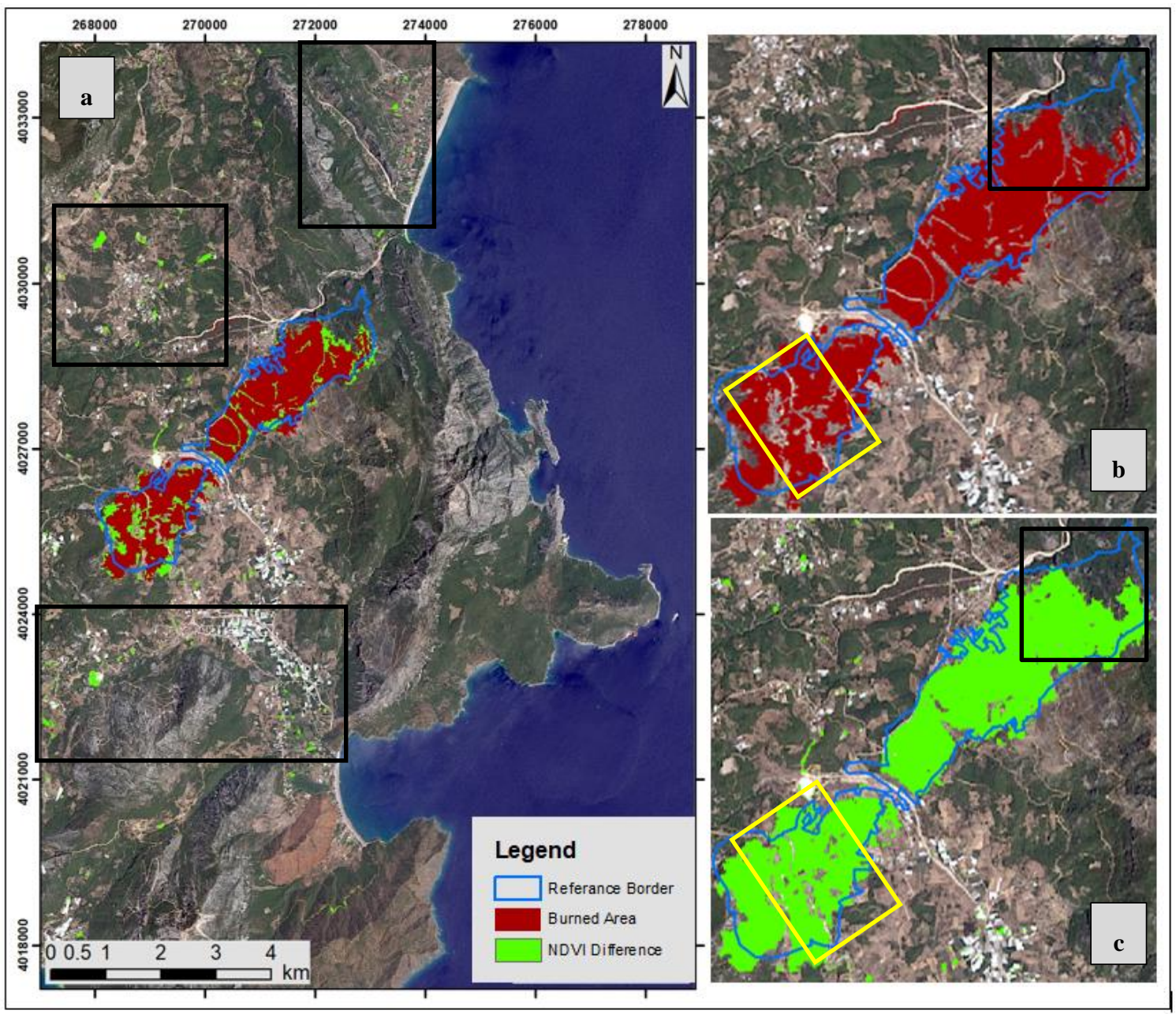

Figure 6. (a): Overlapped of the local map, NDVI difference map and random forest classification results for burning areas, the black rectangle indicates areas with NDVI change similar to burned areas. (b): local burned area border and random forest classification results, the black rectangle shows unchanged vegetation area, the yellow rectangle indicates soil and road area. (c): local burned area border and NDVI difference results, black rectangle shows unchanged vegetation area, the yellow rectangle indicates soil and road area. 
The NDVI difference map was generated for interpretation of the results. Pre-event (22 June 2016) NDVI map was extracted from the post-event (8 July 2016) NDVI map for generating NDVI difference map (dNDVI). Figure 6 shows the local authority burned area border as a blue line, dNDVI results as green color and random forest classification results as red color. According to Figure 6a, the results of dNDVI and random forest classification results largely overlapped, while the local authority map shows a wider area than both. Although there is no change in plant cover in the areas indicated by black rectangles in figures $6 \mathrm{~b}$ and $6 \mathrm{c}$, these areas are shown as a burned area in the local map. Local authority burned area boundary shows a more general area. While the local map showed a general area, the results of the dNDVI map and the random forest classification did not reveal the soil and road areas as burned areas which shows a yellow rectangle in figure $6 \mathrm{~b}$ and figure $6 \mathrm{c}$. These results explain where the difference area in Table 4 came from.

Forest fires are disasters that directly affect the forest cover. If the burning area is below the forest and there is no change in the forest, mapping it from optical satellite images is not possible. In figure $6 \mathrm{~b}$, the area shown by the black rectangle was not mapped with applied method in this study, because forest cover has not changed. On the other hand, change detection analysis made by one attribute such as NDVI, other surfaces could be extracted as burned area. For example, Areas which are shown by the black rectangle in figure 6a has similar NDVI change with the burned areas. In addition, pre-event and postevent images are not always easy to access. Therefore, classification algorithms such as random forests, which can use many attributes over a single image, are very important. In this study, the random forest algorithm was applied at the local scale, but this study can be applied to larger areas.

\section{CONCLUSION}

The rapid development of remote sensing technologies has prompted users to look for ways to develop powerful and effective alternatives at the point of delivery analysis. In recent years, fast and efficient classification algorithms have been developed for classifying data in complex structures, particularly in the classification of satellite images for the production of thematic maps. Among these methods, machine learning algorithm called random forest algorithm is proposed as an effective classification algorithm which is used to solve many application problems including the problem of classification of remotely sensed images.

In this study, a random forest algorithm searched for potential burned areas. 18 variables were used (10 spectral indexes, mean values of 6 bands, brightness, and maximum difference), taken from the literature, to identify burned areas. Since the random forest algorithm randomly selects variables in the classification process, it can be seen that each experiment could benefit from using different variables. As a result of the classification process, the burned areas were determined with a high level of accuracy. The study was carried out in two burned forest areas, on the same date. The Kumluca area was used as training data and the Adrasan area as test data. The results of the classification process show that this method could be used for identifying burned areas with the high accuracy. Future studies will be based on to use the method on the higher resolution images such as Sentinel -2 which provide free data to improve the field of use.

\section{REFERENCES}

Adams, M. A. (2013). Mega-fires, tipping points and ecosystem services: Managing forests and woodlands in an uncertain future. Forest Ecology and Management, 294, 250-261.

Banko, G. (1998). A review of assessing the accuracy of classifications of remotely sensed data and of methods including remote sensing data in forest inventory.

Barbosa, P. M., Grégoire, J.-M., \& Pereira, J. M. C. (1999). An algorithm for extracting burned areas from time series of AVHRR GAC data applied at a continental scale. Remote Sensing of Environment, 69(3), 253-263.

Bastarrika, A., Chuvieco, E., \& Martín, M. P. (2011). Mapping burned areas from Landsat TM/ETM+ data with a two-phase algorithm: Balancing omission and commission errors. Remote Sensing of Environment, 115(4), 1003-1012.

Benz, U. C., Hofmann, P., Willhauck, G., Lingenfelder, I., \& Heynen, M. (2004). Multi-resolution, objectoriented fuzzy analysis of remote sensing data for GISready information. ISPRS Journal of photogrammetry and remote sensing, 58(3), 239-258.

Biau, G., Devroye, L., \& Lugosi, G. (2008). Consistency of random forests and other averaging classifiers. Journal of Machine Learning Research, 9(Sep), 2015-2033.

Breiman, L. (1996). Bagging predictors. Machine learning, 24(2), 123-140.

Breiman, L. (2001). Random forests. Machine learning, 45(1), 5-32.

Chen, G., He, Y., De Santis, A., Li, G., Cobb, R., \& Meentemeyer, R. K. (2017). Assessing the impact of emerging forest disease on wildfire using Landsat and KOMPSAT-2 data. Remote Sensing of Environment, 195, 218-229.

Chen, W., Li, X., Wang, Y., Chen, G., \& Liu, S. (2014). Forested landslide detection using LiDAR data and the random forest algorithm: A case study of the Three Gorges, China. Remote Sensing of Environment, 152, 291-301.

Chuvieco, E., Martin, M. P., \& Palacios, A. (2002). Assessment of different spectral indices in the red-nearinfrared spectral domain for burned land discrimination. International Journal of Remote Sensing, 23(23), 51035110. 
Dey, V., Zhang, Y., \& Zhong, M. (2010). A review on image segmentation techniques with remote sensing perspective: na.

Díaz-Uriarte, R., \& De Andres, S. A. (2006). Gene selection and classification of microarray data using random forest. BMC bioinformatics, 7(1), 3 .

Dillon, G. K., Holden, Z. A., Morgan, P., Crimmins, M. A., Heyerdahl, E. K., \& Luce, C. H. (2011). Both topography and climate affected forest and woodland burn severity in two regions of the western US, 1984 to 2006. Ecosphere, 2(12), 1-33.

Dimitriou, A., Mantakas, G., \& Kouvelis, S. (2001). An analysis of key issues that underlie forest fires and shape subsequent fire management strategies in 12 countries in the Mediterranean basin. Final report prepared by Alcyon for WWF Mediterranean Programme Office and IUCN.

Epting, J., Verbyla, D., \& Sorbel, B. (2005). Evaluation of remotely sensed indices for assessing burn severity in interior Alaska using Landsat TM and ETM+. Remote Sensing of Environment, 96(3), 328-339.

Escuin, S., Navarro, R., \& Fernandez, P. (2008). Fire severity assessment by using NBR (Normalized Burn Ratio) and NDVI (Normalized Difference Vegetation Index) derived from LANDSAT TM/ETM images. International Journal of Remote Sensing, 29(4), 10531073.

Estoque, R. C., Murayama, Y., \& Akiyama, C. M. (2015). Pixel-based and object-based classifications using highand medium-spatial-resolution imageries in the urban and suburban landscapes. Geocarto International, 30(10), 1113-1129.

Filippidis, E., \& Mitsopoulos, I. (2004). Mapping forest fire risk zones based on historical fire data in Mount Olympus, Greece, using geographical information systems. WIT Transactions on Ecology and the Environment, 77.

Flannigan, M. D., \& Haar, T. V. (1986). Forest fire monitoring using NOAA satellite AVHRR. Canadian Journal of Forest Research, 16(5), 975-982.

Fraser, R., Li, Z., \& Cihlar, J. (2000). Hotspot and NDVI differencing synergy (HANDS): A new technique for burned area mapping over boreal forest. Remote Sensing of Environment, 74(3), 362-376.

Gao, Y., Mas, J. F., Kerle, N., \& Pacheco, J. A. N. (2011). Optimal region growing segmentation and its effect on classification accuracy. International Journal of Remote Sensing, 32(13), 3747-3763.

Giglio, L., Loboda, T., Roy, D. P., Quayle, B., \& Justice, C. O. (2009). An active-fire based burned area mapping algorithm for the MODIS sensor. Remote Sensing of Environment, 113(2), 408-420.

Gilbertson, J. K., Kemp, J., \& van Niekerk, A. (2017). Effect of pan-sharpening multi-temporal Landsat 8 imagery for crop type differentiation using different classification techniques. Computers and Electronics in Agriculture, 134, 151-159.

Gislason, P. O., Benediktsson, J. A., \& Sveinsson, J. R. (2006). Random forests for land cover classification. Pattern Recognition Letters, 27(4), 294-300.

Ham, J., Chen, Y., Crawford, M. M., \& Ghosh, J. (2005). Investigation of the random forest framework for classification of hyperspectral data. IEEE Transactions on Geoscience and Remote Sensing, 43(3), 492-501.

Hernandez, C., Drobinski, P., \& Turquety, S. (2015). How much does weather control fire size and intensity in the Mediterranean region? Annales Geophysicae, 33(7), 931-939.

Holden, Z. A., Morgan, P., \& Evans, J. S. (2009). A predictive model of burn severity based on 20-year satellite-inferred burn severity data in a large southwestern US wilderness area. Forest Ecology and Management, 258(11), 2399-2406.

Huete, A., Didan, K., Miura, T., Rodriguez, E. P., Gao, X., \& Ferreira, L. G. (2002). Overview of the radiometric and biophysical performance of the MODIS vegetation indices. Remote Sensing of Environment, 83(1), 195-213.

Huete, A. R. (1988). A soil-adjusted vegetation index (SAVI). Remote Sensing of Environment, 25(3), 295309.

Ishwaran, H. (2007). Variable importance in binary regression trees and forests. Electronic Journal of Statistics, 1, 519-537.

Key, C., \& Benson, N. (2006). Landscape assessment: ground measure of severity, the Composite Burn Index. Pages LA8-LA15 in DC Lutes, editor. FIREMON: Fire Effects Monitoring and Inventory System. USDA Forest Service, Rocky Mountain Research Station, Fort Collins, Colorado, USA.

Kuhn, M., \& Johnson, K. (2013). Applied predictive modeling (Vol. 26): Springer.

Loboda, T., O'neal, K., \& Csiszar, I. (2007). Regionally adaptable dNBR-based algorithm for burned area mapping from MODIS data. Remote Sensing of Environment, 109(4), 429-442.

Louppe, G., Wehenkel, L., Sutera, A., \& Geurts, P. (2013). Understanding variable importances in forests of randomized trees. Paper presented at the Advances in neural information processing systems.

Meinshausen, N. (2006). Quantile regression forests. Journal of Machine Learning Research, 7(Jun), 983-999.

Meng, R., Wu, J., Schwager, K. L., Zhao, F., Dennison, P. E., Cook, B. D., . . Serbin, S. P. (2017). Using high spatial resolution satellite imagery to map forest burn severity across spatial scales in a Pine Barrens ecosystem. Remote Sensing of Environment, 191, 95-109. 
Mitrakis, N. E., Mallinis, G., Koutsias, N., \& Theocharis, J. B. (2012). Burned area mapping in Mediterranean environment using medium-resolution multi-spectral data and a neuro-fuzzy classifier. International Journal of Image and Data Fusion, 3(4), 299-318.

Neyisci, T., Sirin, G., Bas, M. N., \& Saribasak, H. (2016). Antalya - kumluca ve adrasan orman yanginlari hakkinda rapor.

Palandjian, D., Gitas, I. Z., \& Wright, R. (2009). Burned area mapping and post-fire impact assessment in the Kassandra peninsula (Greece) using Landsat TM and Quickbird data. Geocarto International, 24(3), 193-205.

Pereira, J. M. (1999). A comparative evaluation of NOAA/AVHRR vegetation indexes for burned surface detection and mapping. IEEE Transactions on Geoscience and Remote Sensing, 37(1), 217-226.

Petropoulos, G. P., Kontoes, C., \& Keramitsoglou, I. (2011). Burnt area delineation from a uni-temporal perspective based on Landsat TM imagery classification using support vector machines. International Journal of Applied Earth Observation and Geoinformation, 13(1), 70-80.

Petropoulos, G. P., Vadrevu, K. P., Xanthopoulos, G., Karantounias, G., \& Scholze, M. (2010). A comparison of spectral angle mapper and artificial neural network classifiers combined with Landsat TM imagery analysis for obtaining burnt area mapping. Sensors, 10(3), 1967 1985.

Phiri, D., \& Morgenroth, J. (2017). Developments in Landsat Land Cover Classification Methods: A Review. Remote Sensing, 9(9).

Pinty, B., \& Verstraete, M. (1992). GEMI: a non-linear index to monitor global vegetation from satellites. Plant ecology, 101(1), 15-20.

Ramo, R., \& Chuvieco, E. (2017). Developing a Random Forest Algorithm for MODIS Global Burned Area Classification. Remote Sensing, 9(11), 1193.
Schepers, L., Haest, B., Veraverbeke, S., Spanhove, T., Vanden Borre, J., \& Goossens, R. (2014). Burned area detection and burn severity assessment of a heathland fire in Belgium using airborne imaging spectroscopy (APEX). Remote Sensing, 6(3), 1803-1826.

Smith, A., Drake, N., Wooster, M., Hudak, A., Holden, Z., \& Gibbons, C. (2007). Production of Landsat ETM+ reference imagery of burned areas within Southern African savannahs: comparison of methods and application to MODIS. International Journal of Remote Sensing, 28(12), 2753-2775.

Stumpf, A., \& Kerle, N. (2011). Object-oriented mapping of landslides using Random Forests. Remote Sensing of Environment, 115(10), 2564-2577.

Trigg, S., \& Flasse, S. (2001). An evaluation of different bi-spectral spaces for discriminating burned shrubsavannah. International Journal of Remote Sensing, 22(13), 2641-2647.

Tucker, C. J. (1979). Red and photographic infrared linear combinations for monitoring vegetation. Remote Sensing of Environment, 8(2), 127-150.

Vallejo, V. R., Arianoutsou, M., \& Moreira, F. (2012). Fire ecology and post-fire restoration approaches in Southern European forest types. In Post-fire management and restoration of southern European forests (pp. 93119): Springer.

Varamesh, S., Hosseini, S. M., \& Rahimzadegan, M. (2017). Comparison of Conventional and Advanced Classification Approaches by Landsat- 8 Imagery. Applied Ecology and Environmental Research, 15(3), 1407-1416.

Wilson, E. H., \& Sader, S. A. (2002). Detection of forest harvest type using multiple dates of Landsat TM imagery. Remote Sensing of Environment, 80(3), 385-396.

Zhang, Y. (2002). A new automatic approach for effectively fusing Landsat 7 as well as IKONOS images. Paper presented at the Geoscience and Remote Sensing Symposium, 2002. IGARSS'02. 2002 IEEE International. 